\title{
ANTECEDENTES DO RELACIONAMENTO E DA PERFORMANCE EM EMPRESAS DA CADEIA DE SUPRIMENTOS: ESTRUTURAÇÃO E APLICAÇÃO DE MODELOS DE EQUAÇÕES ESTRUTURAIS
}

THE FORERUNNERS OF RELATIONSHIP AND PERFORMANCE IN SUPPLY CHAIN COMPANIES: STRUCTURING AND APPLYING STRUCTURAL EQUATIONS

\section{RESUMO}

O relacionamento entre empresas constitui importante questão na gestão de cadeias de suprimentos. Vários aspectos relacionados ao fluxo e à troca de informações ao longo da cadeia são considerados como tendo influência decisiva no sucesso desse relacionamento. O objetivo central deste trabalho foi estruturar e testar alguns modelos de vínculos entre aspectos dessa natureza com o desempenho e com o relacionamento comprador-fornecedor na cadeia de suprimentos. Foram investigados aspectos pertinentes à comunicação e ao uso de tecnologia da informação no relacionamento entre as empresas. A relação do desempenho com o relacionamento também foi investigada. A pesquisa realizada foi calcada em dados empíricos apurados por meio da modelagem de equações estruturais. Os resultados mostram que alguns aspectos contribuem de forma significativa para o sucesso do relacionamento, enquanto outros, que a priori seriam considerados importantes, não evidenciam contribuição.

\section{Valeriana Cunha}

Professora da Faculdade de Gestão e Negócios, Universidade Federal de Uberlândia - MG, Brasil valeriana_cunha@uol.com.br

\section{Ronaldo Zwicker}

Professor da Faculdade de Economia Administração e Contabilidade, Universidade de São Paulo - SP, Brasil rzwicker@usp.br

Recebido em 23.10.2007. Aprovado em 17.10.2008

Avaliado pelo sistema double blind review

Editor Científico: Henrique Luiz Côrrea

\begin{abstract}
The relationship between companies is an important issue in the management of supply chains. Several aspects relating to the flow and exchange of information along the chain are considered as having a decisive influence on the success of this relationship. The main objective of this work was to structure and test models that link aspects of this nature with performance and the purchaser-supplier relationship in the supply chain. Aspects relevant to communication and the use of IT in relationships between companies were investigated. The importance of performance in this relationship was also investigated. The research was based on empirical data obtained by means of structural equation modeling. The results show that some aspects contribute in a significant way to the success of this relationship while others that, a priori, are considered important make no contribution
\end{abstract}

PALAVRAS-CHAVE Relacionamento, empresas, cadeia de suprimentos, comunicação, tecnologia da informação. KEYWORDS Relationship, companies, supply chain, communication, information technology. 


\section{INTRODUÇÃo}

Existem vários aspectos que podem influenciar o relacionamento e a performance das empresas. Morgan e Hunt (1994) destacam o comprometimento como influenciador do relacionamento entre empresas. Parsons (2002), além de destacar o comprometimento, inclui o fator confiança e a definição de objetivos comuns como ingredientes críticos para determinar o sucesso de um relacionamento. Os estudos de Hormozi (2001) apontam que a agilidade e rapidez na operação são influenciadoras da performance. Ezingeard e outros (2005) e Smith (2000) destacam que a acurácia das informações transacionadas na cadeia de suprimentos é decisiva para a performance das cadeias de suprimentos. Vários pesquisadores (GALT e DALE, 1991; GRAHAM e outros, 1994; LAMBERT e outros, 1996; LASCELLES e DALE, 1989; MCHUGH e outros., 2003; MCLAREN e outros, 2004; MOHR e NEVIN, 1990; MOHR e SPEKMAN, 1994; NEWMAN e RHEE, 1990; PRAHINSKI, 2001) abordam a necessidade de uma boa comunicação entre compradores e fornecedores para que o relacionamento entre eles seja bem sucedido e para que suas performances sejam aprimoradas. Isso é conseguido por meio de uma comunicação eficaz, feedbacks periódicos entre as partes, compartilhamento de informações entre a empresa compradora e seus fornecedores, qualidade das informaçoes transacionadas, entre outros fatores. Outras pesquisas (BYRD e DAVIDSON, 2003; LAFRAMBOISE e REYES, 2005; LARSON e outros, 2005; PETERSEN e outros, 2005; WALTON e MARUCHECK, 1997) mostram que o uso de alguns sistemas de gestão pode influenciar o relacionamento entre as empresas e suas performances, à medida que substituem ou eliminam esforços humanos ineficientes, auxiliam o processo de tomada de decisão e reduzem a variabilidade das decisões e das informações entre as empresas da cadeia.

Atualmente, em um ambiente de negócios com competição tão acirrada, torna-se cada vez mais importante aprimorar o desempenho das empresas nas cadeias de suprimentos e o relacionamento entre tais empresas. Dessa forma, conhecer os fatores que influenciam a performance e o relacionamento é um ponto crucial para a área em estudo.

Esta pesquisa analisa dois grupos de aspectos antecedentes do relacionamento e da performance: aspectos relacionados à comunicação e aspectos relacionados ao uso de tecnologia da informação (TI). Esses dois grupos de antecedentes foram escolhidos por terem o propósito de garantir o correto fluxo de informações entre os membros da cadeia de suprimentos. Vários autores, den- tre eles Chopra e Meindl (2004), afirmam que o fluxo de informações afeta profundamente cada parte da cadeia. Dessa forma, esses dois antecedentes foram considerados de suma importância para o bom funcionamento das cadeias de suprimentos.

O objetivo do trabalho é estruturar e testar modelos para investigar influências diretas e indiretas que a comunicação e a tecnologia da informação exercem no relacionamento comprador-fornecedor e na performance das empresas, bem como a influência do próprio relacionamento na performance das empresas envolvidas.

A modelagem de equações estruturais (structural equation modeling - SEM) foi utilizada para averiguar as possíveis relações causais nos modelos considerados. Segundo Prahinski e Benton (2004), quando há várias relações causais no modelo, a modelagem de equações estruturais é considerada a metodologia mais apropriada, pois tal abordagem permite testar simultaneamente as várias relações para cada um dos modelos propostos. Pesquisas mais recentes relacionadas à comunicação e ao relacionamento na cadeia de suprimentos tendem a se apoiar na modelagem de equações estruturais para a apuração dos modelos propostos. Entretanto, não se detectou o uso da mesma abordagem com modelos que contemplem a influência do uso da tecnologia da informação. Apesar de existir vasta literatura relacionada à utilização de sistemas de TI no gerenciamento de cadeias de suprimentos, percebe-se uma carência de modelos que avaliem os impactos desses sistemas na gestão das cadeias (BYRD e DAVIDSON, 2003).

Este artigo está dividido da seguinte maneira: após esta seção introdutória, contempla uma seção de revisão de literatura, abordando os principais aspectos do gerenciamento da cadeia de suprimentos e alguns antecedentes do relacionamento e da performance das empresas. A terceira seção traz o modelo de pesquisa com as hipóteses testadas. Além disso, apresenta a população-alvo do estudo e suas características. A seguir são apresentadas as questões relacionadas ao desenvolvimento da pesquisa, bem como os resultados encontrados. Finalmente, nas considerações finais, apresentam-se as contribuições e limitações da pesquisa.

\section{GESTÃO DA CADEIA DE SUPRIMENTOS}

Segundo Chopra e Meindl (2004), uma cadeia de suprimentos consiste em todas as partes envolvidas, direta ou indiretamente, em atender as requisições dos clientes. A organização da cadeia envolve inúmeras atividades, $\mathrm{e}$ 
inclui desde o recebimento da solicitação do cliente até a conclusão do atendimento. Os dois fluxos da cadeia de suprimentos mais comumente citados na literatura (CHRISTOPHER, 1998; LEE e BILLINGTON, 1995; LEWIS e TALALAYEVSKY, 1997; NEW E PAYNE, 1995; SWAMINATHAN e outros, 1998) são os fluxos de produtos e informações, mas alguns autores (CHOPRA e MEINDL, 2004; FIALA, 2005; MARQUEZ e outros., 2001) incluem também o fluxo de recursos financeiros. No âmbito do fluxo de informações, dois elementos têm destaque: a comunicação e os recursos que apóiam essa comunicação. Esses elementos são brevemente tratados a seguir, juntamente com os objetos sobre os quais eles possivelmente têm interferência causal: o relacionamento comprador-fornecedor e a performance dos envolvidos.

O relacionamento comprador-fornecedor pode ser considerado como o conjunto de estratégias interorganizacionais empregadas pelas empresas compradoras e fornecedoras em suas negociações. Os autores em geral tratam desse relacionamento considerando diferentes atributos para caracterizá-lo. Para Prahinski (2001), a relação comprador-fornecedor é definida como a percepção do fornecedor acerca do comportamento da firma compradora no âmbito de alguns atributos: o comprometimento da empresa compradora, a sua cooperação e os seus acordos operacionais. Já para Maloni e Benton (2000), o relacionamento comprador-fornecedor é composto por cinco atributos: comprometimento, confiança, conflitos, cooperação e resolução de conflitos. Na pesquisa realizada, optou-se pela abordagem comportamental de Maloni e Benton (2000), pois ela oferece, em princípio, a oportunidade de testar a pertinência de mais atributos no relacionamento das empresas estudadas.

O termo "performance" é bastante abrangente. Na literatura, são encontrados diversos tipos de indicadores utilizados para mensurá-la. Byrd e Davidson (2003) avaliaram três indicadores para mensurar a performance da empresa: o ROI (return on investment), o ROE (return on equity) e o market share da empresa, comparados com os dos competidores mais próximos. Nos modelos de Prahinski (2001), a performance do fornecedor foi definida como um indicador de sucesso competitivo mensurado pela qualidade dos produtos, desempenho na entrega, preço, agilidade de resposta a solicitações de mudanças e oferta de serviços de suporte. Tan e outros (1998) utilizaram como indicadores de performance: lucro, market share, nível de estoque, produtividade e satisfação do cliente final. Jayaram e outros (1999) observam que há um grupo de indicadores de performance baseados no tempo. Os indicadores mais comuns desse grupo são: rapidez da entrega, tempo de desenvolvimento de novos produtos, confiabilidade da entrega, tempo de introdução de novos produtos e tempo de fabricação. Outro indicador de performance é o nível de serviço prestado na cadeia de suprimentos. Um exemplo é o on time, in full (OTIF), que mensura o percentual de entregas realizadas dentro do prazo acordado e com os itens constantes do pedido todos corretamente entregues. Nesta pesquisa, os indicadores utilizados para mensurar a performance financeira foram: preço e prazo de pagamento. Já a performance operacional foi mensurada pelos seguintes indicadores: rapidez na entrega, pontualidade na entrega (on time), perfeição de atendimento do pedido (in full), qualidade dos principais produtos (do fornecedor) ou das embalagens (do distribuidor ou comprador) e mix de produtos. Os itens prazo de pagamento e mix de produtos foram sugeridos no pré-teste da pesquisa. Os outros itens foram considerados por serem os mais citados na literatura como indicadores de desempenho utilizados por empresas de cadeias de suprimentos semelhantes à da pesquisa.

Dois grupos de antecedentes do relacionamento e da performance foram considerados na pesquisa: fatores relacionados à comunicação e fatores relacionados ao uso de tecnologia da informação (TI). Esses dois grupos de antecedentes foram escolhidos com base no argumento de autores como Sanders (2005), Mohr e Nevin (1990), e Mohr e Spekman (1994), de sua possível importância para garantir o correto fluxo de informações entre os membros da cadeia de suprimentos.

Conforme Mohr e Nevin (1990), a comunicação pode ser considerada como a cola que mantém um canal de distribuição unido. A comunicação possui várias facetas que, combinadas, determinam a estratégia da comunicação. Algumas das facetas da comunicação são a periodicidade, o conteúdo compartilhado (tipo de informação), o meio ou canal de comunicação (pessoal, telefone, fax, e-mail, sistemas informatizados), aspectos da comunicação geral (formalidade, regras e procedimentos no processo de comunicação) e o feedback (comunicação bidirecional). São considerados nos modelos estudados na presente pesquisa: a periodicidade, o conteúdo compartilhado, a comunicação geral e o feedback. Tais variáveis foram escolhidas depois de diversas entrevistas com integrantes da Empresa Alfa, que as julgaram as mais importantes no que tange ao relacionamento da empresa com seus fornecedores.

Segundo Sanders (2005), a tecnologia da informação possibilita que os compradores e fornecedores compartilhem grandes volumes de informações operacionais e estratégicas. Zhao e outrsos (2001) destacam que a 
tecnologia e o foco na informação estão sendo cada vez mais vistos como preditores-chave do desempenho das empresas nas cadeias de suprimentos. Para a construção dos modelos, procurou-se abranger os sistemas de gestão mais comumente usados nas empresas e citados na literatura, considerando os três níveis propostos por Pedroso (2002): nível transacional, nível de apoio à tomada de decisão e nível de colaboração.

\section{MODELO DE PESQUISA}

O presente trabalho caracteriza-se como uma pesquisa tipo survey. Segundo Roesch (2005), quando o propósito da pesquisa é medir relações de associação ou causa-efeito entre variáveis, recomenda-se utilizar o enfoque da pesquisa quantitativa. Dadas as várias relações causais a serem testadas na presente pesquisa, considerou-se como mais indicada a utilização da modelagem de equações estruturais. Essa abordagem permitiu testar simultaneamente as várias relações para cada um dos modelos propostos.
Na Figura 1 apresenta-se o modelo conceitual inicial relativo à dimensão comunicação, incluindo-se a designação das hipóteses testadas. O modelo conceitual relativo à dimensão tecnologia da informação segue o mesmo padrão, diferenciando-se apenas pelo fato de as variáveis exógenas serem relacionadas à dimensão TI e não mais à dimensão comunicação.

O Quadro 1 consolida as hipóteses da pesquisa. Cada hipótese ou grupo de hipóteses relaciona-se a um constructo ou à relação entre dois constructos. A influência de cada faceta da comunicação foi avaliada em modelos separados por meio das hipóteses $\mathrm{H}_{3 \mathrm{a}}, \mathrm{H}_{3 \mathrm{~b}}, \mathrm{H}_{3 \mathrm{c}}, \mathrm{H}_{3 \mathrm{~d}}, \mathrm{H}_{4 \mathrm{a}}$, $\mathrm{H}_{4 \mathrm{~b}}, \mathrm{H}_{4 \mathrm{c}}, \mathrm{H}_{4 \mathrm{~d}}$. A influência de cada tipo de sistema de informações também foi avaliada separadamente, conforme hipóteses $\mathrm{H}_{6 \mathrm{a}}, \mathrm{H}_{6 \mathrm{~b}}, \mathrm{H}_{6 \mathrm{c}}, \mathrm{H}_{6 \mathrm{~d}}, \mathrm{H}_{7 \mathrm{a}}, \mathrm{H}_{7 \mathrm{~b}}, \mathrm{H}_{7 \mathrm{c}}, \mathrm{H}_{7 \mathrm{~d}}$.

Todos os constructos dos modelos relativos tanto à comunicação quanto à tecnologia de informação passaram por um processo de caracterização e purificação que provocou mudanças nos modelos dessa proposta inicial. Os procedimentos adotados nesse sentido são discutidos na próxima seção.

Figura 1 - Modelo conceitual da dimensão comunicação

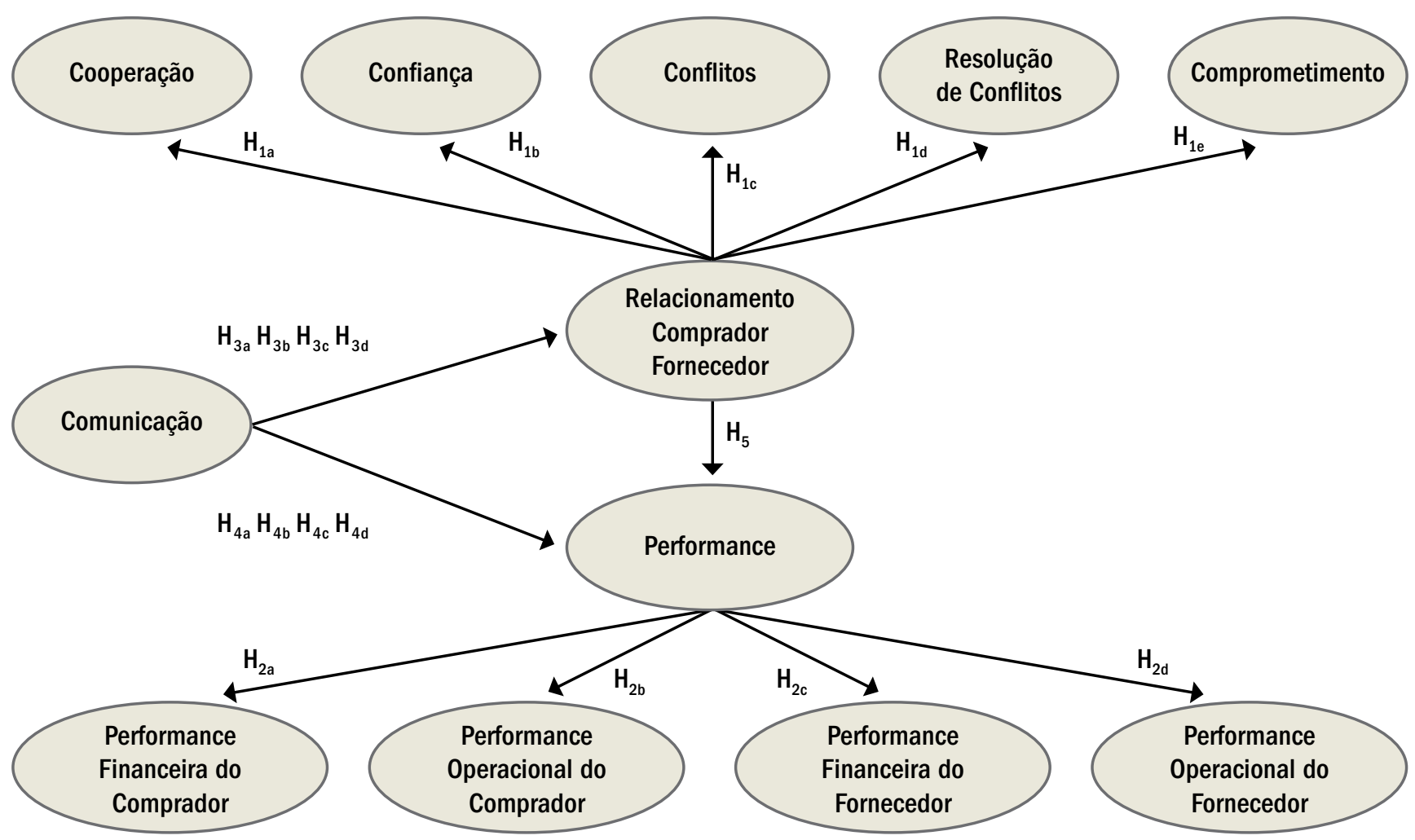


A população-alvo desta pesquisa compreendeu todos os fornecedores ativos da Empresa Alfa, uma distribuidora de medicamentos e produtos de higiene e beleza com sede na cidade de São Paulo. Na época da coleta de dados, a Empresa Alfa negociava com aproximadamente 130 fornecedores e atuava em todo o território nacional, tendo como clientes farmácias, drogarias, supermercados e mercados. Foram utilizadas as técnicas de entrevista e questionário para coleta de dados primários. No questionário foi utilizada a escala Likert com sete pontos. O questionário completo pode ser obtido por solicitação aos autores. Atingiu-se uma taxa de resposta de aproximadamente $80 \%$, com 103 empresas respondentes predominantemente industriais, multinacionais e de grande porte.

\section{Quadro 1 - Hipóteses da pesquisa}

\section{CONSTRUCTOS E} RELAÇÕES

\begin{tabular}{l} 
Relacionamento \\
Comprador-Fornecedor \\
\hline Performance \\
\hline Relação entre \\
Comunicação e \\
Relacionamento \\
Comprador-Fornecedor
\end{tabular}

$\mathrm{H}_{1}$ : 0 Relacionamento Comprador-Fornecedor é um constructo formado por:

$\mathrm{H}_{1 \mathrm{a}}:$ Cooperação

$\mathrm{H}_{1 \mathrm{~b}}$ : Confiança

$\mathrm{H}_{1}$ : : Conflitos

$\mathrm{H}_{1 \mathrm{~d}}$ : Resolução de Conflitos

$\mathrm{H}_{1 \mathrm{e}}$ : Comprometimento

$\mathrm{H}_{2}:$ A Performance é um constructo constituído por:

$\mathrm{H}_{2 \mathrm{a}}$ : Performance Financeira do Comprador

$\mathrm{H}_{2 \mathrm{~b}}$ : Performance Operacional do Comprador

$\mathrm{H}_{2 \mathrm{c}}$ : Performance Financeira do Fornecedor

$\mathrm{H}_{2 \mathrm{~d}}$ : Performance Operacional do Fornecedor

$\mathrm{H}_{3 \mathrm{a}}$ : A Periodicidade influencia positivamente o Relacionamento Comprador-Fornecedor

$\mathrm{H}_{3 b}$ : A Comunicação Geral influencia positivamente o Relacionamento Comprador-Fornecedor

$\mathrm{H}_{3 \mathrm{c}}$ : 0 Feedback influencia positivamente o Relacionamento Comprador-Fornecedor

$\mathrm{H}_{3 \mathrm{~d}}$ : 0 Conteúdo Compartilhado influencia positivamente o Relacionamento Comprador-Fornecedor

\section{Relação entre \\ Comunicação e \\ Performance}

Relação entre Performance e Relacionamento

Comprador-Fornecedor

Relação entre Tecnologia

da Informação e

Relacionamento

Comprador-Fornecedor

Relação entre ecnologia da Informação e Performance

$\mathrm{H}_{4}$ : A Periodicidade influencia positivamente a Performance

$\mathrm{H}_{4 b}$ : A Comunicação Geral influencia positivamente a Performance

$\mathrm{H}_{4 \mathrm{c}}: 0$ Feedback influencia positivamente a Performance

$\mathrm{H}_{4 \mathrm{~d}}: 0$ Conteúdo Compartilhado influencia positivamente a Performance

$\mathrm{H}_{5}$ : 0 Relacionamento Comprador-Fornecedor influencia positivamente a Performance

$\mathrm{H}_{6 a}: 0$ EDI influencia positivamente o Relacionamento Comprador-Fornecedor

$\mathrm{H}_{66}$ : Os Sistemas de Gestão Transacionais influenciam positivamente o Relacionamento CompradorFornecedor

$\mathrm{H}_{6 c}$ : Os Sistemas de Gestão de Apoio à Tomada de Decisão influenciam positivamente o Relacionamento Comprador-Fornecedor

$\mathrm{H}_{6 \mathrm{~d}}$ : Os Sistemas Colaborativos influenciam positivamente o Relacionamento Comprador-Fornecedor

$\mathrm{H}_{7 \mathrm{a}}: 0 \mathrm{EDI}$ influencia positivamente a Performance

$\mathrm{H}_{76}$ : Os Sistemas de Gestão Transacionais influenciam positivamente a Performance

$\mathrm{H}_{7 c}$ : Os Sistemas de Gestão de Apoio à Tomada de Decisão influenciam positivamente a Performance

$\mathrm{H}_{7 \mathrm{~d}}:$ Os Sistemas Colaborativos influenciam positivamente a Performance 


\section{DESENVOLVIMENTO DA PESQUISA}

Nesta seção são apresentadas as questões referentes à preparação e tratamento dos dados, à caracterização dos indicadores e à purificação do modelo de medida.

\section{Preparação e tratamento dos dados}

Todos os indicadores foram avaliados a fim de se verificar se definem adequadamente o constructo a que estavam inicialmente vinculados e se há necessidade de eliminar algum item. O processo de eliminação de itens evoluiu em etapas. Primeiramente foram avaliadas as correlações entre os itens em um mesmo contructo e retirados aqueles que apresentaram baixa correlação. Feito isso, utilizando-se o software SPSS (Statistical Package for the Social Sciences), fez-se uma análise fatorial exploratória com os itens restantes visando analisar quais deles se mantêm e como se agrupam, a fim de se examinar a estrutura da inter-relação entre um grande número de variáveis e definir um conjunto de dimensões ou fatores subjacentes que agrupam variáveis comuns (HAIR e outros, 1998).

Para eliminar itens de acordo com as cargas fatoriais foi utilizada a mesma abordagem empregada por Prahinski (2001). Essa autora considerou três situações: itens com cargas fatoriais abaixo de um valor mínimo em todos os fatores; itens com cargas fatoriais acima do valor mínimo em mais de um fator; e itens com cargas fatoriais acima do valor mínimo em um fator diferente do que foi estabelecido a priori. Segundo a autora, caso o item se enquadre em uma das três condições descritas, deverá ser desconsiderado. No estudo assinalado foi considerado o valor mínimo de 0,30 para as cargas fatoriais. No presente trabalho, em vez de utilizar a regra de bolso proposta por vários autores de que cargas fatoriais acima de 0,30 são consideradas aceitáveis, neste estudo optou-se por utilizar o valor mínimo de carga fatorial de acordo com o tamanho da amostra. De acordo com esse encaminhamento, segundo Hair e outros (1998), para um nível de significância de 0,05, a carga fatorial (para $\mathrm{n}=100$ ) deve ser de, no mínimo, 0,55. Observe-se que, nesse caso, cargas de valor 0,30 só seriam significativas para amostras com $n \geq 350$.

Após o processo de eliminação dos itens para os modelos de comunicação, os fatores considerados explicam $78,84 \%$ dos dados originais. A perda de informação foi de $21,16 \%$. Para os modelos de tecnologia da informação, os fatores considerados explicam $80,83 \%$ dos dados originais. A perda de informação foi de $19,17 \%$.

\section{Caracterização dos indicadores}

Os indicadores foram avaliados quanto à normalidade, li- nearidade e colinearidade. A normalidade univariada foi testada para cada um dos itens do constructo por meio de dois testes: Kolmogorov-Smirnov e Shapiro-Wilk Além disso, foram feitos os testes de normalidade multivariada para todos os constructos e foi calculado o índice de curtose multivariada relativa. Não foi comprovada normalidade multivariada nos constructos, mesmo com a transformação das variáveis em escores normais (normal scores), com exceção dos constructos "performance financeira do comprador" e "performance financeira do fornecedor", que se aproximaram da distribuição normal. Assim como Prado (2004), a perspectiva da linearidade foi verificada por avaliação de gráficos de dispersão e busca de relações que possam configurar aspectos não lineares (via SPSS). Nesse caso não puderam ser confirmados fortes traços de não linearidade nos gráficos. Pode-se observar apenas que os dados ficaram bastante dispersos, o que é comum acontecer em pesquisas de percepção e atitude. Para Mattar (2001), a formação de uma atitude é resultado de crenças, reflexos condicionados, fixações, julgamentos, estereótipos, experiências, exposição a comunicações persuasivas, trocas de informações e experiências com outros indivíduos. A dispersão dos dados analisados indica que as respostas obtidas na pesquisa refletem atitudes bastante diversificadas dos respondentes acerca dos itens abordados. Isso pode ter ocorrido devido ao fato de os respondentes terem perfis distintos, atuarem em regiões diferentes, comercializarem produtos bastante diversificados e possuírem níveis de exigência diferentes. Além disso, foram utilizadas duas medidas comuns para avaliar a colinearidade entre pares de variáveis e entre múltiplas variáveis: a tolerância e o seu inverso, denominado fator de inflação da variância (variance inflation factor). Detectouse que algumas variáveis deveriam ser excluídas do modelo (uma delas é comum aos modelos de comunicação e TI e as outras são variáveis dos modelos de TI).

\section{Purificação do modelo de medida}

De acordo com a abordagem de dois passos de Anderson e Gerbing (1998), primeiramente se fez a análise do modelo de medida, que detalha as relações entre as variáveis observáveis (itens) e as variáveis latentes ou constructos hipotéticos (fatores). Isso foi feito antes de se ajustar um modelo de equação estrutural. A segunda parte é o modelo de equação estrutural, que detalha as relações estruturais preestabelecidas pela teoria ou pesquisa anterior, entre os constructos hipotéticos. Segundo O'Leary-Kelly e Vokurka (1998), o processo de validação do instrumento tem três passos: validade do conteúdo, validade do constructo e validade nomológica. No presente trabalho, a revisão da 
literatura, as entrevistas com profissionais da área em estudo e o pré-teste constituíram a base para garantir a validade de conteúdo. Para garantir a validade do constructo foram avaliados três componentes essenciais: unidimensionalidade, validade convergente e discriminante (por meio de análise fatorial confirmatória, utilizando o software Lisrel (linear structural relations)), e confiabilidade (por meio do Alpha de Cronbach calculado pelo SPSS). Por meio da unidimensionalidade, garante-se que um conjunto de indicadores empíricos está relacionado a apenas um constructo. A confiabilidade e a validade convergente e discriminante dizem respeito ao grau de erro da medida. Finalmente, a validade nomológica é a própria validação do modelo estrutural. Sendo assim, o próximo passo foi testar simultaneamente as relações inicialmente estabelecidas entre os fatores. Os modelos testados, juntamente com as análises acerca de cada um deles, estão apresentados na seção seguinte.

\section{DISCUSSÃO DOS RESULTADOS}

O fato de a distribuição de quase todas as variáveis não apresentar normalidade multivariada torna inapropriada a utilização do método de máxima verossimilhança. Dessa forma, para estimar os coeficientes que representam as relações entre as variáveis, utilizou-se o método DWLS (diagonally weighted least squares). O DWLS tem distribuição assintótica livre, sendo, portanto, mais indicado quando não se consegue confirmar a pressuposição de normalidade/multinormalidade. Flora e Curran (2004) defendem o uso do DWLS para modelos médios a grandes, com escalas ordinais e amostras moderadas a pequenas. No trabalho de Wang (2005), a estimação pelo método DWLS com matriz de correlação policórica mostrou ser a abordagem mais apropriada para a análise de dados ordinais não normais. Dessa forma, no presente trabalho o método de estimação DWLS também foi associado ao uso da matriz de correlação policórica.

\section{Teste de hipóteses}

Ao longo da apuração dos resultados, quatro dos cinco constructos do Relacionamento Comprador-Fornecedor Foram descartados (Confiança, Cooperação, Conflitos e Resolução de Conflitos). O único constructo relacionado ao Relacionamento Comprador-Fornecedor que não foi retirado foi o constructo Comprometimento. Ainda assim, alguns itens que formavam o constructo foram eliminados, itens esses representantes do comprometimento da empresa compradora. Sendo assim, as hipóteses $\mathrm{H}_{1 \mathrm{a}}, \mathrm{H}_{1 \mathrm{~b}}, \mathrm{H}_{1 \mathrm{c}}, \mathrm{H}_{1 \mathrm{~d}}$ e $\mathrm{H}_{1 \mathrm{e}}$ foram consideradas inconclusivas, pois não foram testadas em nenhum dos modelos, e o constructo passou a ser representado exclusivamente pelo Comprometimento do Fornecedor. Vale lembrar que a utilização dos cinco constructos se baseou na pesquisa de Maloni e Benton (2000). Em nenhuma outra referência bibliográfica pesquisada foram utilizados os cinco constructos exatamente como descritos pelos citados autores, para representar o Relacionamento Comprador-Fornecedor. Além disso, a maioria das referências considera vários compradores e vários fornecedores para avaliar a relação entre as empresas. Nesta pesquisa foram considerados diversos fornecedores e apenas uma empresa compradora. Dessa forma, não se localizam argumentos contrários para, no caso dessa forma de relação específica, representar o Relacionamento Comprador-Fornecedor apenas pelo Comprometimento do Fornecedor. Também foram consideradas inconclusivas as duas hipóteses relacionadas à Comunicação Geral $\left(\mathrm{H}_{3 \mathrm{~b}} \mathrm{e} \mathrm{H}_{4 \mathrm{~b}}\right)$, pois este constructo também foi eliminado na fase de validação e purificação das escalas. Além dessas, as hipóteses referentes ao constructo Sistemas de Gestão para o Apoio à Tomada de Decisão $\left(\mathrm{H}_{6 c}\right.$ e $\left.\mathrm{H}_{7 \mathrm{c}}\right)$ também não foram testadas devido à alta multicolinearidade entre todos os itens do constructo e, portanto, foram consideradas inconclusivas.

Em relação ao outro constructo de segunda ordem (Performance), em todos os modelos se verificou a aceitação da hipótese de que ele é formado pelos constructos de primeira ordem: Performance Financeira do Fornecedor, Performance Operacional do Fornecedor, Performance Financeira do Comprador e Performance Operacional do Comprador. Entretanto, é importante ressaltar que, para os modelos de Tecnologia da Informação (EDI, Sistemas de Gestão Transacionais, Sistemas de Gestão Colaborativos), após a aplicação das análises fatoriais, a Performance do Comprador não foi separada nos dois constructos: Financeiro e Operacional. Os itens referentes à Performance Financeira e à Performance Operacional permaneceram juntos em um único constructo denominado Performance do Comprador. Os Quadros 2 e 3 apresentam as hipóteses estabelecidas no início do trabalho e os resultados dos testes delas. É importante ressaltar que essas hipóteses se referem aos efeitos diretos entre os constructos. Além disso, o teste de hipótese considera apenas a significância estatística da relação. Portanto, além de analisar os testes de hipóteses, é importante considerar os efeitos totais (composição dos efeitos diretos e indiretos), bem como a força das relações. Essas questões são tratadas a seguir. 


\section{ARTIGOS - ANTECEDENTES DO RELACIONAMENTO E DA PERFORMANCE EM EMPRESAS DA CADEIA DE SUPRIMENTOS: ESTRUTURAÇÃO E APLICAÇÃO DE MODELOS DE EQUAÇÕES ESTRUTURAIS}

\section{Modelos estruturais}

A apuração dos modelos estruturais foi realizada utilizando-se o software Lisrel. Além da verificação da validade das relações propostas (testes das hipóteses inicialmente estabelecidas), avaliaram-se os efeitos totais entre as variáveis de cada modelo, além de se analisarem alguns índices de ajuste dos modelos. É importante ressaltar que os modelos agora apresentados diferem dos mode- los conceituais inicialmente estipulados devido aos processos de validação e purificação das escalas. A Figura 2 sintetiza os modelos de Comunicação testados para as variáveis exógenas: Feedback, Compartilhamento de Informações, Periodicidade da Comunicação por Meios Convencionais, Periodicidade da Comunicação por Fax/Carta e Periodicidade da Comunicação por Meio Eletrônico.

Quadro 2 - Resultados dos testes das hipóteses $\mathrm{H}_{1 \mathrm{a}}$ a $\mathrm{H}_{3 \mathrm{~d}}$
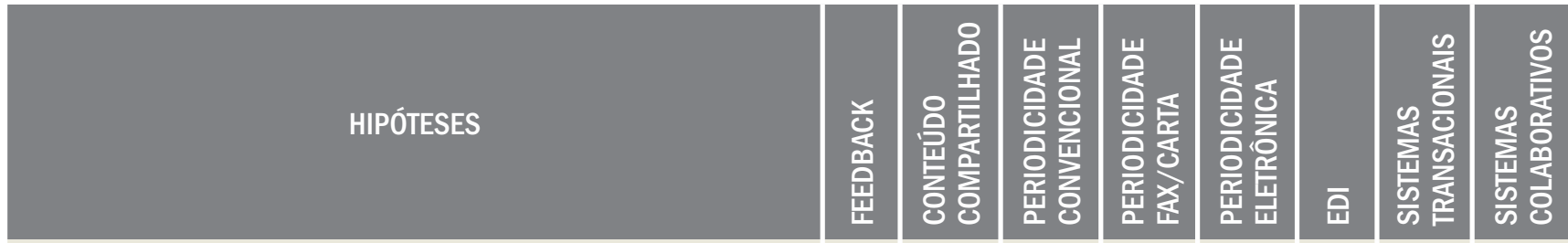

$\mathrm{H}_{1 \mathrm{a}}: 0$ Relacionamento Comprador-Fornecedor é um constructo multidimensional e influencia a Cooperação

$\mathrm{H}_{1 \mathrm{~b}}$ : 0 Relacionamento Comprador-Fornecedor é um constructo multidimensional e influencia a Confiança

$\mathrm{H}_{1 \mathrm{c}}$ : 0 Relacionamento Comprador-Fornecedor é um constructo multidimensional e influencia os Conflitos

$\mathrm{H}_{1 \mathrm{~d}}: 0$ Relacionamento Comprador-Fornecedor é construc to multidimensional e influencia a Resolução de Conflitos

$\mathrm{H}_{1}: \mathrm{e}$ Relacionamento Comprador-Fornecedor é um cons-tructo multidimensional e influencia o Comprometimento

$\mathrm{H}_{2 \mathrm{a}}$ : A Performance é um constructo constituído pela Performance Financeira do Comprador

$\mathrm{H}_{2 \mathrm{~b}}$ : A Performance é um constructo constituído pela Performance Operacional do Comprador

$\mathrm{H}_{2 \mathrm{c}}$ : A Performance é um constructo constituído pela Performance Financeira do Fornecedor

$\mathrm{H}_{2 \mathrm{~d}}$ : A Performance é um constructo constituído pela Performance Operacional do Fornecedor

$\mathrm{H}_{3 \mathrm{a}}$ : A Periodicidade influencia positivamente o Relacionamento Comprador-Fornecedor

$\mathrm{H}_{3 \mathrm{~b}}$ : A Comunicação Geral influencia positivamente 0

Relacionamento Comprador-Fornecedor

$\mathrm{H}_{3 \mathrm{c}}$ : 0 Feedback influencia positivamente o Relacionamento

Comprador-Fornecedor

$\mathrm{H}_{3 \mathrm{~d}}: 0$ Conteúdo Compartilhado influencia positivamente 0 Relacionamento Comprador-Fornecedor

$\begin{array}{llclcl}\text { V1 } & \text { Verificada a 0,01 } & \text { V5 } & \text { Verificada a 0,05 } & \text { V10 } & \text { Verificada a 0,10 } \\ \text { R } & \text { Rejeitada } & \text { I } & \text { Inconclusiva } & - & \text { Não testada neste modelo }\end{array}$


A Figura 3 sintetiza os modelos de Tecnologia da Informação testados para as variáveis exógenas: EDI, Sistemas de Gestão Transacionais e Sistemas de Gestão Colaborativos.

\section{Modelos de comunicação}

As variáveis exógenas avaliadas nos modelos de comunicação foram Feedback, Compartilhamento de Informações e Periodicidade da Comunicação. Da análise dos modelos estruturais pode-se concluir que o Feedback exerce forte influência direta e positiva no Relacionamento Comprador-Fornecedor. Prahinski (2001) também concluiu que o Feedback influencia positivamente o Relacionamento Comprador-Fornecedor. Anderson e Weitz (1992) concluíram que a comunicação aberta e bidirecional leva ao aumento do comprometimento na ca-

Quadro 3 - Resultados dos testes das hipóteses $\mathrm{H}_{4 \mathrm{a}}$ a $\mathrm{H}_{7 \mathrm{~d}}$

\begin{tabular}{|c|c|c|c|c|c|c|c|c|}
\hline HIPÓTESES & 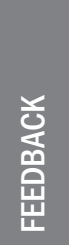 & 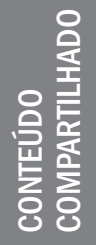 & 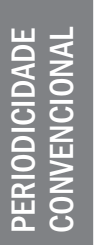 & 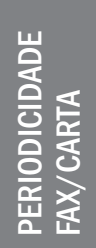 & 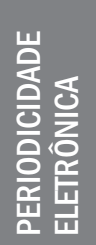 & 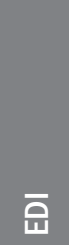 & 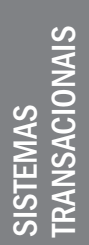 & 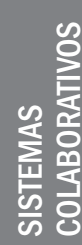 \\
\hline $\mathrm{H}_{4 \mathrm{a}}$ : A Periodicidade influencia positivamente a Performance & - & - & $\mathrm{R}$ & V5 & V10 & - & - & - \\
\hline $\mathrm{H}_{4 \mathrm{~b}}$ : A Comunicação Geral influencia positivamente a Performance & I & I & I & I & I & I & I & I \\
\hline $\mathrm{H}_{4 \mathrm{c}}: 0$ Feedback influencia positivamente a Performance & $\mathrm{R}$ & - & - & - & -- & - & - & - \\
\hline $\begin{array}{l}\mathrm{H}_{4 \mathrm{~d}}: 0 \text { Conteúdo Compartilhado influencia positivamente a } \\
\text { Performance }\end{array}$ & - & V5 & - & - & - & - & - & - \\
\hline $\begin{array}{l}\mathrm{H}_{5}: 0 \text { Relacionamento Comprador-Fornecedor influencia } \\
\text { positivamente a Performance }\end{array}$ & V1 & V10 & V1 & V1 & V1 & V1 & V1 & V1 \\
\hline $\begin{array}{l}\mathrm{H}_{6 \mathrm{a}}: 0 \mathrm{EDI} \text { influencia positivamente o Relacionamento Comprador- } \\
\text { Fornecedor }\end{array}$ & - & - & - & - & - & $\mathrm{R}$ & - & - \\
\hline $\begin{array}{l}\mathrm{H}_{66}: \text { Os Sistemas de Gestão Transacionais influenciam } \\
\text { positivamente o Relacionamento Comprador-Fornecedor }\end{array}$ & - & - & - & - & - & - & $\mathrm{R}$ & - \\
\hline $\begin{array}{l}\mathrm{H}_{6 c}: \text { Os Sistemas de Apoio à Decisão influenciam positivamente } 0 \\
\text { Relacionamento Comprador-Fornecedor }\end{array}$ & 1 & 1 & I & I & I & I & I & I \\
\hline $\begin{array}{l}\mathrm{H}_{6 \mathrm{~d}}: \text { Os Sistemas Colaborativos influenciam positivamente } 0 \\
\text { Relacionamento Comprador-Fornecedor }\end{array}$ & - & - & - & - & - & - & - & $\mathrm{R}$ \\
\hline $\mathrm{H}_{7 \mathrm{a}}: 0 \mathrm{EDI}$ influencia positivamente a Performance & - & - & - & - & - & $\mathrm{R}$ & - & - \\
\hline $\begin{array}{l}\mathrm{H}_{76} \text { : Os Sistemas de Gestão Transacionais influenciam } \\
\text { positivamente a Performance }\end{array}$ & - & - & - & - & - & - & $\mathrm{R}$ & - \\
\hline $\begin{array}{l}\mathrm{H}_{7 c}: \text { Os Sistemas de Gestão de Apoio à Tomada de Decisão } \\
\text { influenciam positivamente a Performance }\end{array}$ & 1 & 1 & 1 & I & I & 1 & I & I \\
\hline $\begin{array}{l}\mathrm{H}_{\mathrm{7d}}: \text { Os Sistemas Colaborativos influenciam positivamente a } \\
\text { Performance }\end{array}$ & - & - & - & - & - & - & - & V5 \\
\hline $\begin{array}{llclc}\text { V1 } & \text { Verificada a 0,01 } & \text { V5 } & \text { Verificada a 0,05 } & \text { V10 } \\
\text { R } & \text { Rejeitada } & \text { I } & \text { Inconclusiva } & -\end{array}$ & 0 te & 0 , & delo & & & & & \\
\hline
\end{tabular}


deia de suprimentos tanto da empresa fabricante quanto da empresa distribuidora. Mohr e outros (1996) concluíram que a bidirecionalidade (que estes autores consideram como componente da comunicação colaborativa) tem um efeito mais forte e positivo no comprometimento dos membros da cadeia de suprimentos sob baixos níveis de controle do que sob altos níveis de controle. Da análise também se conclui que o Feedback não apresentou influência direta na Performance. No trabalho de Prahinski (2001), a hipótese de que o Feedback influencia direta e positivamente a Performance do Fornecedor também foi rejeitada. A análise foi complementada verificandose a influência indireta do Feedback na Performance, e a conclusão é de que o Feedback exerce influência indireta positiva mediana na Performance conjunta da empresa compradora e fornecedora (via Relacionamento Comprador-Fornecedor) e fraca nas subdimensões da Performance.

Em relação ao Compartilhamento de Informações, tanto da empresa compradora para seus fornecedores como o inverso, a principal conclusão foi de que o Compartilhamento de Informações exerce forte influência direta e positiva no Relacionamento CompradorFornecedor. Cannon e Perreault (1999) concluíram que, nas relações onde o Compartilhamento de Informações se mostrou mais significativo, a firma compradora reportou ter um alto grau de confiança no fornecedor. A confiança era uma das dimensões do modelo conceitual a ser testado na presente pesquisa, no entanto tal dimensão foi eliminada no processo de validação das escalas. $\mathrm{O}$ Compartilhamento de Informações também exerce forte influência positiva na Performance (considerando a composição dos efeitos diretos e indiretos, via Relacionamento Comprador-Fornecedor) e baixa influência positiva nas subdimensões da Performance. Walton e Marucheck (1997) concluíram que o compartilhamento de algumas informações, tais como planos de produção e de capacidade, está associado com o aprimoramento do desempenho na entrega. Entretanto, para os mesmos autores, o compartilhamento de previsões pelo comprador ou o compartilhamento de informações de produção pelo fornecedor está associado a baixos níveis de desempenho na entrega.

Mohr e outros (1996) concluíram que a frequência da comunicação influencia o comprometimento dos membros da cadeia de suprimentos, principalmente sob baixos níveis de controle; entretanto, os autores não especificaram o meio utilizado na comunicação. Na presente pesquisa, após a realização da análise fatorial exploratória, a Periodicidade da Comunicação foi dividida em: periodicidade por meios convencionais, por fax/carta e por meio eletrônico. Os resultados mostram que a Periodicidade da Comunicação Convencional entre empresa compradora e seus fornecedores exerce baixa influência direta e positiva no Relacionamento Comprador-Fornecedor. Por outro lado, a Periodicidade da Comunicação Convencional não

Figura 2 - Modelo final comunicação

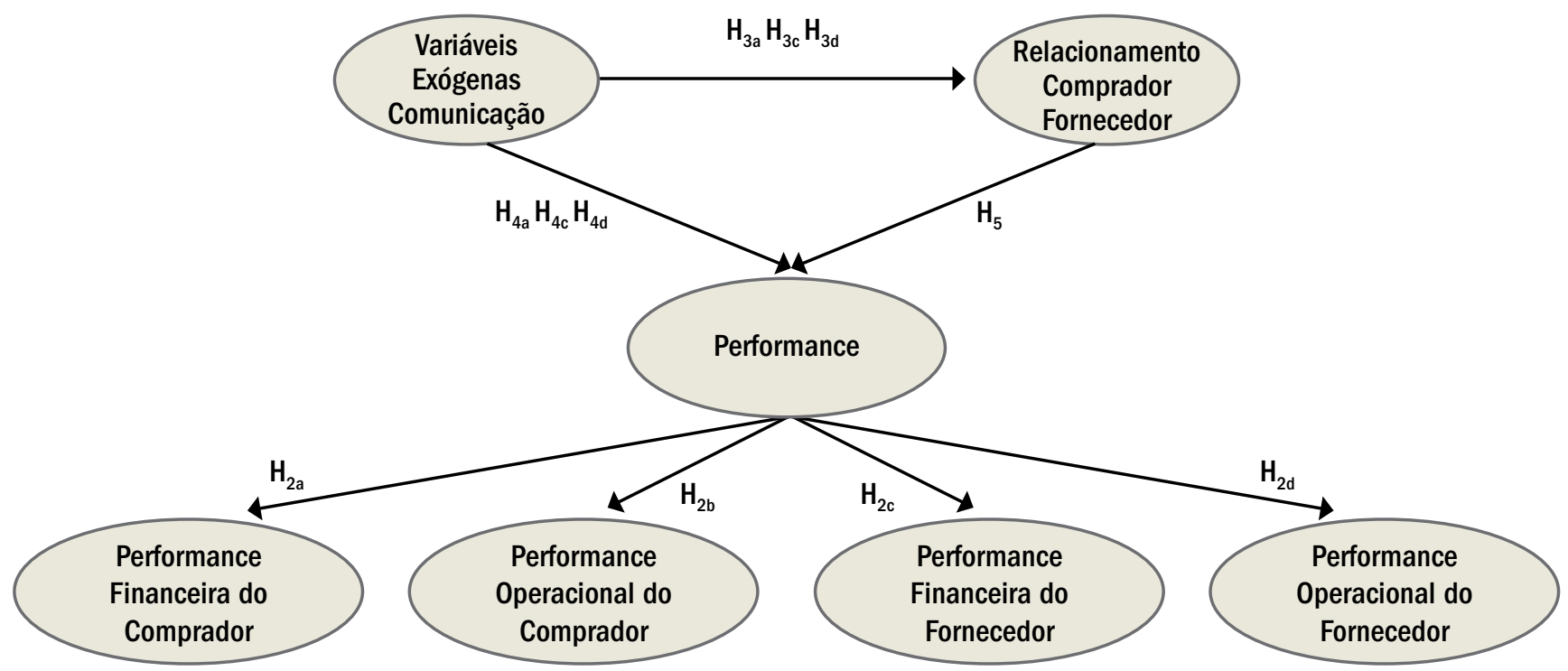


influencia diretamente a Performance e exerce uma baixa influência indireta (via Relacionamento CompradorFornecedor) positiva nela. Entretanto, ao analisar a combinação dos efeitos diretos e indiretos, a influência da Periodicidade da Comunicação Convencional na Performance não é estatisticamente significante. Além disso, a Periodicidade da Comunicação Convencional não influencia nenhuma das subdimensões da Performance.

Em relação à Periodicidade da Comunicação por Fax/ Carta entre empresa compradora e seus fornecedores, pode-se dizer que a variável não influencia diretamente o Relacionamento Comprador-Fornecedor. Além disso, a Periodicidade da Comunicação por Fax/Carta exerce baixa influência direta positiva na Performance e não a influencia indiretamente (via Relacionamento Comprador-Fornecedor). Considerando a combinação dos efeitos diretos e indiretos, conclui-se que a influência da Periodicidade da Comunicação por Fax/Carta na Performance não é estatisticamente significante. Também se verificou que a Periodicidade da Comunicação por Fax/Carta exerce uma baixa influência indireta na Performance Financeira do Comprador e na Performance Operacional do Comprador (via Performance). A influência indireta na Performance do Fornecedor (tanto operacional quanto financeira) não se mostrou estatisticamente significante.

Em relação à Periodicidade da Comunicação por Meio Eletrônico entre a empresa compradora e seus fornece- dores, pode-se dizer que não influencia diretamente o Relacionamento Comprador-Fornecedor. Também se concluiu que a Periodicidade da Comunicação por Meio Eletrônico exerce uma baixa influência direta positiva na Performance e não exerce influência indireta nela. Sendo assim, somando os efeitos indiretos (que não são significantes) e diretos, o efeito total da Periodicidade da Comunicação por Meio Eletrônico na Performance não é estatisticamente significante. Por fim, a Periodicidade da Comunicação por Meio Eletrônico não apresentou influência estatisticamente significante em nenhuma das subdimensões da Performance.

\section{Modelos de tecnologia da informação}

As variáveis exógenas avaliadas nos modelos de TI relacionam-se à utilização do EDI, de Sistemas de Gestão Transacionais e de Sistemas de Gestão Colaborativos. O uso do EDI não mostrou influência direta no Relacionamento Comprador-Fornecedor nem na Performance. Vijayasarathy e Robey (1997) concluíram que o uso do EDI aprimora a cooperação entre as partes envolvidas na comunicação. A cooperação era um dos constructos de primeira ordem que representariam o constructo de segunda ordem Relacionamento Comprador-Fornecedor. Entretanto, esta dimensão foi excluída do modelo após o processo de validação das escalas. Além disso, a utilização de EDI não apresentou influência indireta na Performance (via Relacionamento

Figura 3 - Modelo final TI

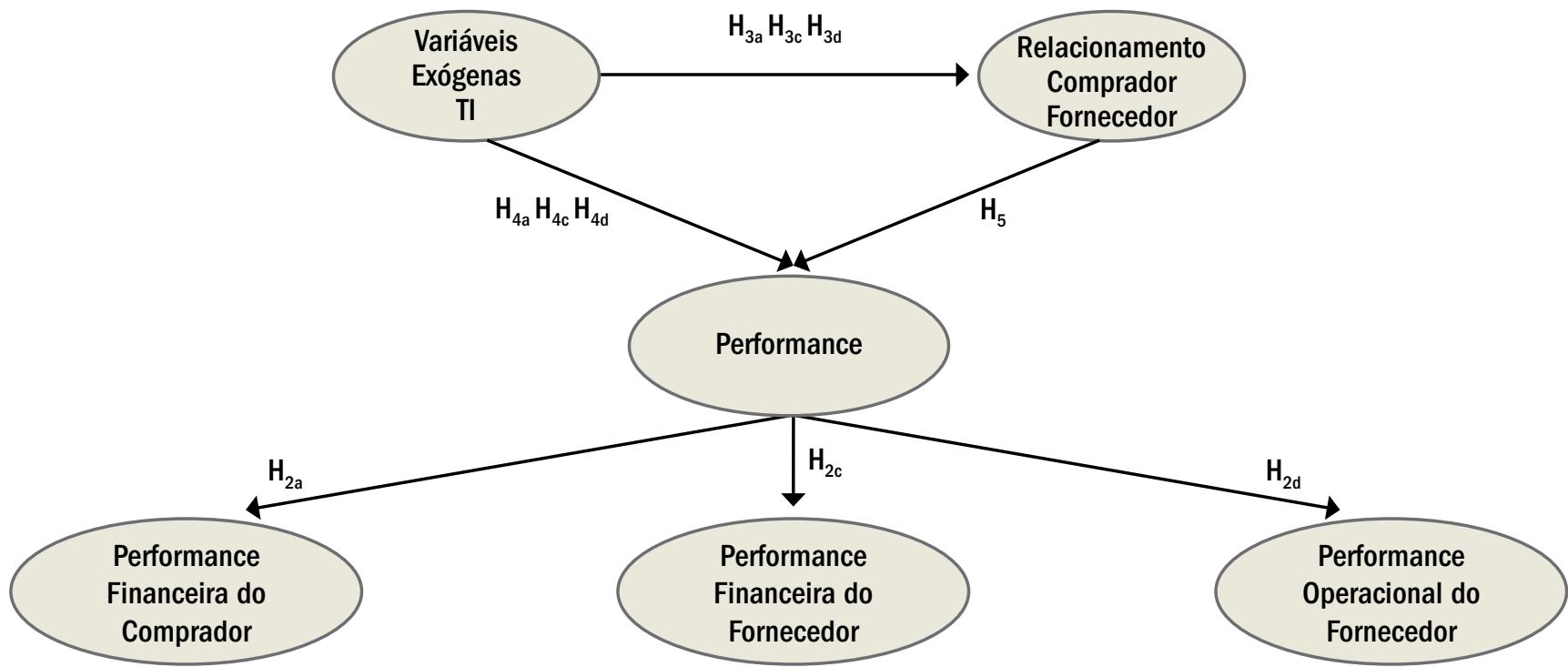


Comprador-Fornecedor) nem nas suas subdimensões. Eventualmente este resultado pode ser considerado compatível com Walton e Marucheck (1997), que concluíram que o tempo que as empresas despedem no uso de EDI com determinado fornecedor influencia negativamente a performance de entrega. Entretanto, os mesmos autores concluíram que o número de fornecedores que usam EDI influencia positivamente a performance de entrega. É preciso lembrar que, na presente pesquisa, o EDI foi mensurado considerando o seu grau de utilização para a troca de informações entre o comprador e o fornecedor. Claramente, o grau de utilização não se refletiu no relacionamento nem na performance.

O uso de Sistemas de Gestão Transacionais foi mensurado de acordo com o grau de utilização de alguns sistemas transacionais por parte da empresa fornecedora. As conclusões sugerem que o uso desse tipo de sistema não influencia diretamente o Relacionamento Comprador-Fornecedor nem a Performance, e também não influencia indiretamente a Performance (via Relacionamento Comprador-Fornecedor) nem as suas subdimensões (via Relacionamento ou via Performance). Laframboise e Reyes (2005) testaram especificamente a influência do uso de ERPs na posição competitiva e performance e concluíram que a utilização desses sistemas influencia a posição competitiva e a performance indiretamente via interação com outros recursos. A influência direta do uso de ERPs na performance não foi comprovada pelos autores.

Em relação ao uso de Sistemas de Gestão Colaborativos, as seguintes conclusões podem ser arroladas: o uso de Sistemas Colaborativos não influencia diretamente o Relacionamento Comprador-Fornecedor, exerce baixa influência positiva direta na Performance e não exerce nela influência indireta. A influência total (somando-se os efeitos diretos e indiretos) dos Sistemas Colaborativos na Performance é baixa. Nas subdimensões da Performance há influência, entretanto ela é ainda mais baixa que na Performance conjunta. Petersen e outros (2005) concluíram que o planejamento colaborativo estruturado e o não estruturado influenciam positivamente a Performance do Fornecedor. Por sua vez, Corsten e Felde (2005) concluíram que quanto maior é o nível de colaboração da empresa compradora com seus fornecedores maior é a performance financeira da empresa compradora. Nenhum desses autores considerou sistemas específicos de gestão colaborativa como se fez na presente pesquisa.

\section{Índices de ajuste dos modelos estruturais}

Segundo Hair e outros (1998), avaliar o ajuste geral dos modelos de equações estruturais não é uma tarefa tão simples e direta como acontece em outras técnicas multivariadas, pois não há um teste estatístico único que melhor descreva a "força" de previsão de um determinado modelo. Dessa forma, pesquisadores tais como Jöreskog e Sörbom (1981); Bentler e Bonett (1980); Tucker e Lewis (1973) desenvolveram algumas medidas que, ao serem usadas conjuntamente, servem para avaliar os resultados sob três perspectivas: ajuste geral, ajuste comparativo ao modelo base e parcimônia do modelo. O uso de múltiplos índices é aconselhável, pois fornece evidência convergente ao ajuste do modelo. De modo geral o ajustamento dos modelos com base nos índices considerados (vide Hair e outros (1998)) foi satisfatório, conforme mostram as medidas arroladas no Quadro 4.

Quadro 4 - Avaliação do ajustamento global dos modelos

\begin{tabular}{|l|c|c|c|c|c|c|c|c|}
\hline \multirow{2}{*}{ MODELOS } & \multicolumn{3}{|c|}{ MEDIDAS ABSOLUTAS } & \multicolumn{3}{c|}{ MEDIDAS INCREMENTAIS } & $(*)$ \\
\cline { 2 - 10 } & $\lambda^{2}$ & GFI & RMSR & RMSEA & NFI & AGFI & NNFI & $\lambda^{2} / \mathrm{GL}$ \\
\hline Feedback & 84,41 & 0,98 & 0,08 & 0,00 & 0,96 & 0,98 & 1,01 & 0,86 \\
\hline Compartilhamento Informações & 141,28 & 0,97 & 0,09 & 0,05 & 0,93 & 0,96 & 0,98 & 1,25 \\
\hline Comunicação Convencional & 219,44 & 0,96 & 0,10 & 0,07 & 0,90 & 0,95 & 0,96 & 1,49 \\
\hline Comunicação por Fax/Carta & 106,65 & 0,98 & 0,09 & 0,03 & 0,94 & 0,97 & 0,99 & 1,09 \\
\hline Comunicação Eletrônica & 211,00 & 0,97 & 0,10 & 0,08 & 0,90 & 0,96 & 0,95 & 1,64 \\
\hline EDI & 85,60 & 0,99 & 0,07 & 0,00 & 0,95 & 0,98 & 1,01 & 0,87 \\
\hline Sistemas Transacionais & 149,51 & 0,99 & 0,07 & 0,01 & 0,94 & 0,99 & 1,00 & 1,02 \\
\hline Sistemas Colaborativos & 107,78 & 0,99 & 0,07 & 0,03 & 0,94 & 0,98 & 0,99 & 1,09 \\
\hline
\end{tabular}

(*) Medida parcimoniosa. 


\section{CONSIDERAÇÕES FINAIS}

Este trabalho apresenta contribuições teóricas a partir das informações encontradas empiricamente. Os dados foram cuidadosamente analisados, seguindo o embasamento metodológico expresso na literatura.

Alguns resultados encontrados são coerentes com pesquisas já realizadas, como, por exemplo, a influência positiva do Feedback no Relacionamento CompradorFornecedor destacada por Prahinski (2001). Outros resultados não seguem a mesma linha proposta por alguns autors na literatura. Espera-se que a pesquisa possa agregar à literatura existente os resultados apresentados sobre a influência da comunicação e do uso de determinados sistemas de gestão no relacionamento e performance das empresas fornecedoras e compradoras.

Do ponto de vista prático, é importante lembrar que a amostra de fornecedores da empresa Alfa examinada na pesquisa pode ser considerada bastante representativa e que os resultados refletem a opinião da maioria dos fornecedores. Especificamente neste caso, as conclusões obtidas podem ser úteis no direcionamento de ações das empresas envolvidas, no sentido do aprimoramento do seu relacionamento. Entre outras coisas, os resultados obtidos deixam evidentes os aspectos que têm ou não significância nesse relacionamento. Conforme Gunasekaran e outros (2004) destacam, é preciso reunir os membros da cadeia de suprimentos para que eles discutam o aprimoramento das relações na cadeia. Eventualmente pesquisas do tipo da que acaba de ser relatada podem ser úteis para despertar o interesse das partes em trabalharem conjuntamente, para intensificar e promover um maior desenvolvimento das suas cadeias de suprimentos.

Do ponto de vista metodológico, a estimação dos parâmetros via DWLS apresenta um diferencial da pesquisa no campo de estudo em relação à maioria dos trabalhos encontrados na literatura, visto que o método não está entre os mais populares métodos de estimação. No entanto, o seu uso para a situação encontrada neste trabalho está bem embasado na literatura e apresentou bons resultados nos modelos testados.

A pesquisa apresenta algumas limitações que precisam ser destacadas. Primeiramente, é preciso lembrar que todas as respostas foram dadas pelas empresas fornecedoras. Como havia perguntas relacionadas aos fornecedores $e$ também à empresa compradora, um ponto de reflexão sobre essa limitação é se os fornecedores respondentes realmente tinham o conhecimento adequado para responder sobre as questões relacionadas à empresa compradora. Por isso, deve-se deixar bem claro que todas as relações causais verificadas refletem opiniões emitidas pelos fornecedores da Empresa Alfa. Além disso, é importante destacar que, mesmo sendo um trabalho quantitativo, há nele bastante subjetividade, pois todas as relações traçadas basearam-se em percepções dos respondentes. A percepção não é algo fácil de mensurar em uma pesquisa em que o contato entre respondente e pesquisador foi pequeno. Essa impessoalidade do trabalho pode levar a interpretações diferentes por parte dos respondentes acerca de uma mesma questão, o que pode resultar em problemas nas respostas utilizadas como base das análises realizadas.

\section{REFERÊNCIAS}

ANDERSON, J. C; GERBING, D. W. Structural equation modeling in practice: a review and recommended two-step approach. Psychological Bulletin, v. 103 , n. 3, p. 411-423, 1998

ANDERSON, E; WEITZ, B. The use of pledges to build and sustain commitment in distribution channels. Journal of Marketing Research, v. 29, n. 1, p. 18-34, 1992.

BENTLER, P. M; BONETT, D. G. Significance tests and goodness-of-fit in the analysis of covariance structures. Psychological Bulletin, v. 88, p. 588-606, 1980

BYRD, T. A; DAVIDSON, N. W. Examining possible antecedents of IT impact on the supply chain and its effect on firm performance. Information \& Management, v. 41, n. 2, p. 243-255, 2003

CANNON, J. P; PERREAULT JR., W. D. Buyer-seller relationships in business markets. Journal of Marketing Research, v. 36, n. 4, p. 439-460, 1999.

CHRISTOPHER, M. Logistics and supply chain management. 2nd ed. New York: Financial Times/Prentice Hall, 1998.

CHOPRA, S; MEINDL, P. Supply chain management: strategy, planning, and operation. 2nd ed. Upper Saddle River: Pearson/Prentice Hall, 2004

CORSTEN, D; FELDE, J. Exploring the performance effects of key-supplier collaboration: an empirical investigation into Swiss buyer-supplier relationships. International Journal of Physical Distribution \& Logistics Management, v. 35, n. 6, p. 445-461, 2005.

EZINGEARD, J.-N. e outros. A model of information assurance benefits EDPACS, v. 32, n. 11, p. 1-16, 2005

FIALA, P. Information sharing in supply chains. The International Journal of Management Science, v. 33, p. 419-423, 2005

FLORA, D. B; CURRAN, P. J. An empirical evaluation of alternative methods of estimation for confirmatory factor analysis with ordinal data Psychological Methods, v. 9, n. 4, p. 466-491, 2004 


\section{ARTIGOS - ANTECEDENTES DO RELACIONAMENTO E DA PERFORMANCE EM EMPRESAS DA CADEIA DE SUPRIMENTOS: ESTRUTURAÇÃO E APLICAÇÃO DE MODELOS DE EQUAÇÕES ESTRUTURAIS}

GALT, J. D. A; DALE, B. G. Supplier development: a British case study. International Journal of Purchasing and Materials Management, v. 27, n. 1, p. 16-22, 1991.

GRAHAM, T. S; DAUGHERTY, P. J; DUDLEY, W. N. The long-term strategic impact of purchasing partnerships. International Journal of Purchasing and Materials Management, v. 30, n. 4, p. 13-19, 1994

GUNASEKARAN, A; PATEL, C; MCGAUGHEY, R. A framework for supply chain performance measurement. International Journal of Production Economics, v. 87, n. 3, p. 333-347, 2004.

HAIRJR., J. F; ANDERSON, R. E; TATHAM, R. L; BLACK, W. C. Multivariate data analysis. 5th ed. Upper Saddle River: Prentice Hall, 1998.

HORMOZI, A. M. Agile manufacturing: the next logical step. Benchmarking, v. 8, n. 2 , p. 132-143, 2001.

JAYARAM, J; VICKERY, S. K; DROGE, C. An empirical study of timebased competition in the North American automotive supplier industry. International Journal of Operations \& Production Management, v. 19, n. 10, p. 1010-1033, 1999

JÖRESKOG, K. G; SÖRBOM, D. LISREL V: Analysis of linear structural relationships by the method of maximum likelihood. Chicago: National Education Resources, 1981.

LAFRAMBOISE, K; REYES, F. Gaining competitive advantage from integrating enterprise resource planning and total quality management. The Journal of Supply Chain Management, p. 49-64, 2005.

LAMBERT, D. M; EMMELHAINZ, M. A; GARDNER, J. T. So you think you want a partner? Marketing Management, v. 5, n. 2, p. 24-40, 1996.

LARSON, P. D; CARR, P; DHARIWAL, K. S. SCM Involving small versus large suppliers: relational exchange and electronic communication media. Journal of Supply Chain Management, v. 41, n. 1, p. 18-29, 2005.

LASCELLES, D. M; DALE, B. G. The buyer-supplier relationship in total quality management. Journal of Purchasing and Materials Management, $\mathrm{v}$. 25 , n. 2, p. 10-19, 1989

LEE, H. L; BILLINGTON, C. The evolution of supply chain management models and practice at Hewlett-Packard. Interfaces, v. 23, n. 5, p. 42-63, 1995

LEWIS, I; TALALAYEVSKY, A. Logistics and information technology: a coordination perspective. Journal of Business Logistics, v. 18, n. 1, p. 141157,1997

MALONI, M; BENTON, W. C. Power influences in the supply chain. Journal of Business Logistics, v. 21, n. 1, p. 49-73, 2000.

MARQUEZ, A. C; OVALLE, O. R; FRAMINAN, J. M. Benefits of the internet for supply chain management: a characterization and simulation study. International Journal of Agile Manufacturing, v. 4, n. 2, p. 25-42, 2001.

MATTAR, F. N. Pesquisa de marketing. São Paulo: Atlas, 2001. 278 p.
MCHUGH, M; HUMPHREYS, P; MCIVOR, R. Buyer-supplier relationships and organizational health. Journal of Supply Chain Management, v. 39, n. 2, p. 15-25, 2003.

MCLAREN, T. S; HEAD, M. M; YUAN, Y. Supply chain management information systems capabilities: an exploratory study of electronics manufacturers. Information Systems and eBusiness Management, v. 2, n. 2, p. 207-222, 2004

MOHR, J. J; FISHER, R. J; NEVIN, J. R. Collaborative communication in interfirm relationships: moderating effects of integration and control Journal of Marketing, v. 60, n. 3, p. 103-115, 1996.

MOHR, J. J; NEVIN, J. R. Communication strategies in marketing channels: a theoretical perspective. Journal of Marketing, v. 54, n. 4, p. 36-51 1990

MOHR, J. J; SPEKMAN, R. Characteristics of partnership success: partnership attributes, communication behavior, and conflict resolution techniques. Strategic Management Journal, v. 15, n. 2, p. 135-152, 1994.

MORGAN, R. M; HUNT, S. D. The commitment-trust theory of relationship marketing. Journal of Marketing, v. 58, n. 3, p. 20-38, 1994.

NEW, S; PAYNE, P. Research frameworks in logistics: three models, seven dinners and a survey. International Journal of Physical Distribution and Logistics Management, v. 25, n. 10, p. 60-77, 1995.

NEWMAN, R. G; RHEE, K. A. A case study of NUMMI and its suppliers International Journal of Purchasing and Materials Management, v. 26, n. 4, p. $15-20,1990$

O'LEARY-KELLY, S. W; VOKURKA, R. J. The empirical assessment of construct validity. Journal of Operations Management, v. 16, n. 4, p. $387-$ 405, 1998

PARSONS, A. L. What determines buyer-seller relationship quality? An investigation from the buyer's perspective. Journal of Supply Chain Management, v. 38, n. 2, p. 4-12, 2002.

PEDROSO, M. C. Um estudo sobre o desenvolvimento das competências em gestão da cadeia de suprimentos. Tese de Doutorado em Engenharia de Produção. Escola Politécnica da Universidade de São Paulo. São Paulo, 2002

PETERSEN, K. J; RAGATZ, G. L; MONCZKA, R. M. An examination of collaborative planning effectiveness and supply chain performance. The Journal of Supply Chain Management, v. 41, n. 2, p. 14-25, 2005.

PRADO, P. H. M. A avaliação do relacionamento sob a ótica do cliente: um estudo em bancos de varejo. Tese de Doutorado em Administração de Empresas. Fundação Getúlio Vargas. São Paulo, 2004

PRAHINSKI, C. Communication Strategies and Supplier Performance Evaluations in an Industrial Supply Chain. Tese de Doutorado em Administração de Empresas. Graduate School of The Ohio State University. Columbus, 2001 


\section{VALERIANA CUNHA · RONALDO ZWICKER}

PRAHINSKI, C; BENTON, W. C. Supplier evaluations: communication strategies to improve supplier performance. Journal of Operations Management, v. 22, n. 1, p. 39-62, 2004.

ROESCH, S. M. A. Projetos de estágio e de pesquisa em administração: guia para estágios, trabalhos de conclusão, dissertações e estudos de caso. 3 a ed. São Paulo: Atlas, 2005.

SANDERS, N. R. IT Alignment in supply chain relationships: a study of supplier benefits. Journal of Supply Chain Management, v. 41, n. 2, p. 4-13, 2005.

SMITH, W. B. Outsourcing supply chain information technology. Logistics Spectrum, v. 34, n. 1, p. 7-9, 2000.

SWAMINATHAN, J. M; SMITH, S. F; SADEH, N. M. Modeling supply chain dynamics: a multiagent approach. Decision Sciences, v. 29, n. 3, p. 607-632, 1998.

TAN, K. C; KANNAN, V. R; HANDFIELD, R. B. Supply chain management: supplier performance and firm performance. International Journal of Purchasing and Materials Management, v. 34, n. 3, p. 2-9, 1998.
TUCKER, L. R; LEWIS, C. A reliability coefficient for maximum likelihood factor analysis. Psychometrika, v. 38, p. 1-10, 1973.

VIJAYASARATHY, L. R; ROBEY, D. The effect of EDI on market channel relationships in retailing. Information \& Management, v. 33, n. 2, p. $73-$ 86,1997

WALTON, S. V; MARUCHECK, A. S. The relationship between ED and supplier reliability. International Journal of Purchasing and Materials Management, 1997.

WANG. W. C. A comparison of Alternative Estimation Methods in Confirmatory Factor Analyses of the General Health Questionnaire Across Four Groups of Australian Immigrants. Dissertação de Mestrado em Estatística Aplicada. Swinburne University of Technology. Lilydale, 2005.

ZHAO, M; DROGE, C; STANK, T. P. The effects of logistics capabilities on firm performance: customer-focused versus information-focused capabilities. Journal of Business Logistics, v. 22, n. 2. p. 91-107, 2001. 